Article

\title{
Effects of Symbol Component on the Identifying of Graphic Symbols from EEG for Young Children with and without Developmental Delays
}

\author{
Chih-Hsiung Huang ${ }^{1}$ and Pei-Jung Lin ${ }^{2, *}$ (]) \\ 1 Department of Early Childhood Education, Nanhua University, Chiayi 622, Taiwan; hk633193@gmail.com \\ 2 Department of Computer Science and Information Engineering, Hungkuang University, \\ Taichung 433, Taiwan \\ * Correspondence: lin.tomato@gmail.com; Tel.: +886-4-26318652
}

Received: 3 February 2019; Accepted: 21 March 2019; Published: 26 March 2019

check for updates

\begin{abstract}
Using Augmentative and Alternative Communication (AAC) to improve the communication skills of children with disabilities is generally supported by both domestic and foreign scholars. Graphic symbols that represent individual words or phrases are often used in conjunction with AAC; however, research on the reading and identifying of AAC graphic symbols is scant. Therefore, this study used electroencephalogram (EEG) to investigate the success rates of identifying AAC graphic symbols and brainwave changes of young children with and without developmental delays. The results revealed that the number of symbol components affected participants' success rates of identifying AAC graphic symbols. The EEG Attention Index between the children with and without developmental delays varied during the test. By contrast, the EEG Relaxation Index exhibited no difference between the children with and without developmental delays. When the participants viewed the single-component animations, the children without developmental delays had a significantly higher Relaxation Index than those with developmental delays did. According to cognitive load theory, the children with developmental delays and low cognitive capacities may feel stressed.
\end{abstract}

Keywords: Augmentative and Alternative Communication (AAC); picture communication symbols (PCS); symbol learning; developmental delays; electroencephalogram (EEG) technology

\section{Introduction}

During the last 20 years, a wide variety of evidence has indicated that augmentative and alternative communication (AAC) can improve the communication skills of children with disabilities [1-3]. Mukhopadhyay \& Nwaogu, [4] stated that AAC exhibits potential advantages to improve language, literacy, and communication skills even for nonspeaking children with intellectual disabilities and is imperative to their learning and communicative competence. Most researchers in AAC studies have employed graphic symbols as vocabulary items to improve the communication skills of children with disabilities; these children have been taught how to express their needs and interact with others through graphic symbols [5-9]. Graphic symbols are considered as the techniques for enhancing efficiency to communicate and primary vocabulary for children with disabilities $[10,11]$. Emms and Gardner [6] argued that, for AAC users, graphic symbols are the most credible alternative vocabulary, and the development of a graphic symbol vocabulary is absolutely critical and fundamental for pediatric AAC users with disabilities. The critical role of graphic symbols in AAC highlights the importance of symbol learning topics in AAC research. Applications of graphic symbols are extensive in both research and teaching practice. Numerous researchers have used graphic symbols to improve 
the communication skills of students with disabilities or mitigate their challenging behavior $[1,10]$. Numerous studies have reported that picture book reading found effective for laying the foundation for children's future literacy capabilities [9,12-14]. It is closely related to language development, print awareness, and emergent literacy [9,12-14]. In addition, graphic symbols are frequently used as a teaching medium and as learning materials in both preschool and special education. Sandall and Schwartz [15] encourage teachers and parents to use pictures and symbols to (a) improve oral instruction comprehension for children with disabilities and (b) promote social interaction and participation between all children with and without disabilities. Therefore, the use and learning of graphic symbols are imperative for young children with and without disabilities. Most studies have argued that graphic symbols can assist children with disabilities in functional competence (e.g., communication, writing, and learning) or improve their communication and literacy skills [6-11]. However, graphic symbols are merely a part of a symbol system, and the so-called symbols refer to objects that stand for or represent referents. Beukelman and Mirenda [5] contended that if symbols characterize the referents, they exhibit a problem of recognizability or specificity. Researchers have also pointed out that many AAC studies have explored the learnability of various graphic symbol systems through the intrinsic characteristics of the systems, and iconicity of symbols has often drawn attention from researchers and practitioners [16-18].

Symbol iconicity refers to any association between a symbol and a referent. The association can be either based on a visual concept or any feature association by the viewer. Published studies have primarily measured symbol iconicity through their transparency and translucency [16]. Symbol transparency refers to the degree to which viewers can directly understand the referent or meaning represented by an individual graphic symbol. Essentially, transparency means the guessability of symbols for viewers without prior training or learning. By contrast, symbol translucency refers to the degree to which viewers can understand or infer the referent or meaning represented by a graphic symbol when some limited descriptive explanation or a contrast object is present. Symbol translucency is also known as the learnability of symbols. Because the iconicity of symbols is one of the key variables that affect symbol learning [19], researchers that have long identified these challenges and have sought solutions proposed an iconicity hypothesis which states that graphic symbols with high iconicity promote symbol learning and comprehension and favor learning outcomes [20]. For years, the iconicity hypothesis has continued to garner attention [18], and learners have been able to easily learn and comprehend graphic symbols with high iconicity. Essentially, graphic symbols that bear a greater resemblance to their referent are more easily learned by individuals [17]. Often, the key challenge of learning AAC applications is whether the learner can quickly associate the graphic symbol iconicity with language and communication functions. Therefore, when communication training or instructional intervention is involved, instructors should select graphic symbols with high transparency and translucency from a variety of symbols and symbol systems to facilitate effective learning outcomes [5].

Most studies on symbol iconicity have compared various graphic symbol systems and reported that symbol systems with high specificity or a variety of symbol elements (e.g., picture communication symbols (PCSs)) yield favorable learning outcomes [16]. Empirical analyses have also revealed that the characteristics of corresponding referents affect the learnability of symbols [21]. Moreover, some studies have verified the iconicity hypothesis and reported that, in addition to favorable learning outcomes yielded by graphic symbols with high iconicity, the complexity of symbols and components are major factors of symbol learning [22]. Although numerous studies have been published on the characteristics and learnability of AAC symbols, few of such studies have been published in Taiwan. In light of this, preliminary studies on AAC symbols and iconicity of color PCS systems commonly used in Taiwan have revealed that the translucency of color PCS systems tended to be high, and nouns had higher translucency than both verbs and descriptors did. In addition, the results confirmed the graphic symbol iconicity hypothesis and demonstrated that PCSs with high translucency favored the participants' learning outcomes [18]. Huang [13] investigated graphic symbol learning; 
the effects of 27 sets of self-compiled verb graphic symbols on the learning outcomes of 40 children with developmental delays and 120 typically developing children were measured. The results revealed that a wide variety of symbol components favored symbol recognition and learning for both children with and without developmental delays. In addition, participants demonstrated significantly higher performance on verb graphic symbols with two, four, and five components than they did on those with a single component in terms of accuracy. Furthermore, children with developmental delays required more time to identify verb graphic symbols with more components.

However, from the cognitive psychology perspective, children should first pay attention to certain content they read to trigger their learning; the cognitive process varies with the time spent on reading pictures or texts [23]. The dual-coding theory believes that multi-element graphic symbols provide more visual clues and stimuli, which can help readers form a coherent mental representation, which is conducive to the recognition and memory of graphic symbols [24]. Some studies support a positive relationship between the number of symbol elements and the degree of recognition $[13,16,19]$. However, cognitive load theory holds the opposite position [25]. It believes that human beings have a limited ability to process information. Excessive information volume can cause readers to overload information. Under the premise of limited working memory capacity, if readers need to encode and organize multiple elements at the same time, it is easy to cause cognitive resources to compete and distract, which is not conducive to the identification and learning of graphic symbols $[25,26]$. Some literature addressing graphic symbol recognition also supports cognitive load theory $[22,27,28]$.

Although most relevant studies $[13,16,17,29]$ have reported that the variety of symbol components affects graphic symbol recognition and learning, the following questions warrant in-depth attention: (a) whether the complexity of graphic symbols (i.e., the number of symbol components) affects children's concentration when they learn or read PCSs composed of various symbol components, (b) whether graphic symbols with numerous components increase or decrease children's reading concentration, and (c) whether graphic symbols with numerous components affect learning outcomes.

Published studies on AAC graphic symbol learning have employed techniques such as video recording, observation of behavior, and computer systems to chronicle the learning reactions of the participants [6]. However, the data collected through the aforementioned methods can only present the participants' responses and reaction times after learning or reading graphic symbols; such data do not demonstrate the learning or reading processes (e.g., the children's concentration) or the association of concentration and reaction time with learning outcomes from reading PCSs and animations composed of various numbers of components. Researchers have focused on this problem for investigations of AAC symbol learning in children. However, conventional behavioral observation and computer documentation cannot demonstrate the children's inner psychological process when they learn AAC symbols. Fortunately, the application of brain research in education has gradually become a trend, and researchers have applied various brain research devices to objectively explore the variance of learning competence. Numerous studies have examined the correlations of electroencephalogram (EEG) data, learning outcomes, and concentration. In recent years, as brainwave-detecting devices have become increasingly lightweight (e.g., Neurosky MindSet and MindWave) and research and development of Brain-Computer Interface (BCI) programming have continued to advance, experts and scholars have increasingly participated in the discipline of brainwave analysis [30,31].

Medical and educational applications of brainwave measurement have emerged because of the association between brainwaves and human consciousness. From the viewpoint of attention theory and cognitive neuroscience, the importance of studying brainwave intensity is to link the level of mental description of information processing in cognitive science to the anatomical level in neuroscience. A brainwave is quantified as a difference between high and low potentials of an electrophysiological signal in the brain. Generally, brainwaves are measured by connecting electrodes to the scalp. The human brain contains an estimated total of 50-100 billion neurons connecting to up to 1 quadrillion synapses, and the brain passes signals through the electric currents generated by these neuron cells [31]. Brainwaves are loosely classified according to frequency range into four categories, 
namely alpha, beta, theta, and delta waves. The alpha wave can be easily detected in a relaxed state (e.g., eyes closed, zoning out, and absent-minded), whereas the beta wave tends to be detected in an alert state (e.g., playing sports and concentrated thinking). These two types of brainwaves are the types of brainwaves most relevant to human beings' conscious activities. One can calculate alpha and beta waves to obtain the numerical values of brain concentration and relaxation.

The present study employed Neurosky EEG devices and systems to investigate the effects and variance of the number of symbol components on AAC graphic symbols identifying for children with and without developmental delays. The two objectives of the present study are listed as follows: (a) to further understand the factors of AAC graphic symbols learning, and (b) to identify the most suitable number of graphic symbol components for children with developmental delays.

\section{Related Work}

The information processing theory emphasizes that learning is an internal psychological activity of human beings; learning is a process of acquiring and utilizing knowledge through sensory awareness, attention, identification, conversion, and memory [32]. Attention is one of the crucial factors of learning outcomes. During the learning process, attention facilitates active learning, comprehension, and familiarity. Humans exhibit mental attention when they are engaged in various activities; mental attention exhibits three aspects, namely selectivity, continuance, and transferability [23]. Learning requires the transmission of signals; the first step of learning is to stimulate the sensory nervous system from some external object, leading to the excitement. During the entire process, once the stimulus disappears, the excitement of the sensory nervous system briefly remains and becomes a signal $[23,32]$. Therefore, one must continuously focus on the topic to be learned to convert it into effective signals processed by the brain [33]. Previous educational research has mainly examined students' attention through explicit behavioral observation and documentation, but the research data have consistently lacked objectivity. From the viewpoint of attention theory and cognitive neuroscience, the importance of studying brainwave intensity is to link the cognitive science concepts of the level of mental description during information processing to the neuroscience concepts of the anatomical level.

After discovering the electric discharge released by the electric eel in the late 19th century, Hans Berger guessed that the same phenomenon would occur in the human body [34]. Subsequently, he recorded the same radio phenomenon in the human skull. Berger documented and measured the tiny discharge process in the human brain. This was the first time in human history that human brainwaves were recorded, and this process Berger named "EEG." Because of the association between brainwaves and human consciousness, medical and educational applications of brainwaves emerged thereafter. Numerous studies have pointed out the close associations of brainwaves with aspects such as stress, job performance, learning outcomes, and attention [34,35]. For example, some studies have stressed that athletes' attention is strongly enhanced in certain brainwave states (e.g., archers can effectively improve their performance by concentrating on aiming) [35]. Regarding learning outcomes, some theories have stated that the rhythms of classical music can change the state of brainwaves so that people can focus on learning and work with considerable efficiency [36]. Some scholars have also analyzed and explored the relationship between brainwaves and learning outcomes. After the statistical data of various attributes had been obtained, e-learning materials were recommended according to the user profile [37]. Brainwaves are the differences between high and low potentials of the electronic signals in the human brain and are generally measured by connecting electrodes to the scalp. Because the brain consists of two cerebral hemispheres, the transmitted signals vary; this phenomenon is known as frontal EEG asymmetry [35]. Brainwaves are loosely classified by frequency range into four categories, namely alpha, beta, theta, and delta waves [35]. The alpha wave can be easily detected in a relaxed state, whereas the beta wave tends to be detected in an alert state (e.g., concentrated thinking). By contrast, the theta and delta waves become prominent in states of low consciousness or unconsciousness (e.g., meditation and deep sleep). Because the theta and delta waves are weak, cluttered, and irregular, the present study did not investigate these two types of waves. 
Regarding the bands of brainwaves, each frequency range exhibits a specific physiological significance. Alpha waves are neural oscillations in the frequency range of approximately $8-13 \mathrm{~Hz}$. According to published studies, zen qigong training effectively relieves stress because high-frequency and calm alpha waves are generated during meditation, indicating a direct relationship between alpha waves and emotions [38]. The decreased energy consumption in the body leads to strong brainwaves, which is the primary reason that scientists have argued that alpha waves benefit learning outcomes. Because alpha waves become prominent during muscle relaxation, when the participant is blinking, when the participant's eyes are closed, or when the participant is asleep, alpha brainwaves are often employed in music therapy [37,39]. Klimesch and his colleagues [40] compared the EEGs of children with and without dyslexia who performed working memory tasks of three difficulty levels. The results revealed that the normal children's high and low alpha waves were more dispersed and selective than those of children with dyslexia. In addition, the normal children employed fewer neural resources (e.g., active high alpha waves) with a high attentional level (i.e., inactive low alpha waves) to complete semantic encoding than the children with dyslexia did. Therefore, alpha wave activity in the frontal lobe can be regarded as an indicator of inefficient information processing in children's working memory. By contrast, beta waves are neural oscillations in the frequency range of $14-30 \mathrm{~Hz}$ that are prominent when people are stressed or focused [41,42]. When human beings exercise, focus on thinking, or are busy or paying attention to something, the brain is in a waking state and the generated brainwaves are known as beta waves. Beta waves are advantageous brainwaves that are prominent in an awake and proactive mental state and are primarily associated with activities in the left hemisphere. A prolonged period in such a mental state leads to body tension. Consequently, the prolonged mental tension drains the brain and makes the person feel tired easily while the physical exhaustion causes anxiety, irritability, and inability to relax. The attention and relaxation parameters examined in the present study were the beta and alpha waves calculated from EEG measurements. Generally, when a participant was in a focused state, the relaxation parameter decreased substantially. By contrast, when a participant was in a relaxed state, the attention parameter did not increase.

Brainwave intensity is a relatively abstract and complex concept and is predominantly obtained by beta wave calculation. Beta waves are classified into two types, namely high and low beta waves. High beta waves are brainwaves with a relatively high frequency $(\geq 30 \mathrm{~Hz})$; the high portion of the beta range overlaps with the low portion of the gamma range. High beta waves become prominent during intensive brain activities and fast information processing. A recent study developed an interface to enable wheelchair control through attentive focus [43]. Another study employed shooting accuracy in archery and coin-stacking height to confirm that human beings' attention in a conscious state far exceeds that in a normal state [44]. Technological advances in medical science have made brainwave research one of the key topics in applied science and technology. Applications of EEG monitoring have become widespread in various disciplines. For example, numerous scholars in the field of design have investigated the effect of lighting color arrangement on brainwaves; this has applied to design e-books and learning materials that increase readers' attention [45]. Moreover, intact phonological processing is crucial for successful literacy acquisition. While learning reading and writing, children should be aware of the sounds of their mother tongue from segmental phonemes as the smallest sound elements to the prosody of supra segmental words and phrases. Phonemic awareness has not only been found to explain individual differences in reading and spelling but also to differentiate typical and literacy-impaired populations (e.g., developmental dyslexia) [46]. In addition, other studies have investigated brainwave differences among individuals with disabilities and examined $\mathrm{BCI}$ applications [31]. In conventional EEGs, the participant is required to wear a head covering that encompasses the entire scalp and collects brainwave signals. Inside the head covering are several electrodes that touch the scalp; the electrodes are coated with conductive adhesives to improve signal collection. Furthermore, the measurement must take place in a specific environment without interference. In recent years, EEG devices have overcome the constraints of conductive adhesives and special locations; brainwaves can now be measured with an EEG headset. Currently, the primary brain 
activities that can be detected by EEGs comprise attention, excitement, and relaxation. The present study investigated the effect of the number of symbol components on AAC graphic symbol learning using EEGs to explore and analyze the attention and relaxation parameters of children with and without developmental delays when learning PCSs with various numbers of components. The findings demonstrate potential factors of PCS learning.

\section{Material and Methods}

The purpose of this study was to investigate the effects of the number of graphic symbol components on the brainwaves of children with and without developmental delays after reading and learning PCSs. Although the research objective was suitable for experimental and quasi-experimental research design, complete random sampling would have been difficult to achieve because the participants were children with developmental delays. In addition, the vast differences of the participants' attributes meant that they could not be randomly assigned to an experimental and a control group. Therefore, this study employed a quasi-experimental design with a pretest and a posttest but without group partition. The independent variable was the number of graphic symbol components, and the dependent variables were the participants' learning outcomes and EEG histories while learning PCSs, including the accuracy of identifying correct graphic symbols and the EEG attention (primarily the beta waves) and relaxation (primarily the alpha waves) parameters.

\subsection{Research Design}

This study recruited children with developmental delays currently attending kindergarten in Taiwan for purposive sampling. The selection criteria for children with developmental delays were as follows: (a) currently attending a public or private kindergarten, (b) experiencing developmental delays certified by a doctor's diagnosis or the Special Education Students Diagnosis and Placement Counseling Committee of a county government, (c) possessing basic receptive language skills (e.g., ability to understand commands and simple sentences), and (d) no other disabilities, such as physical, sensory, and emotional handicaps. In addition, to understand the homogeneity and variance of PCS recognition and learning between children with developmental delays and their peers without developmental delays, the research staff requested that the kindergarten teachers recommend two peer students without special educational needs who frequently interacted with the participating student with developmental delays. The research team visited the kindergarten classes to explain research objectives, implementation methods, expected outcomes, data confidentiality, and ethical compliance to the kindergarten's managers, teachers, parents, and participating children. Finally, after obtaining written consent from the parents and the kindergarten teachers, the research team recruited 17 children with developmental delays and 34 peer students without developmental delays from five kindergarten classes in Taiwan as research participants. The primary disabilities among the 17 children with special needs were cognitive and language developmental delays. The biological ages ranged between 67 and 77 months (or 5.7 and 6.5 years) $(S D=2.88$ months) with a mean age of 72 months (6.0 years). The male-female ratio of the 17 participants with special needs was 9:8. Similarly, the biological ages of the peer students without special needs ranged between 68 and 77 months (or 5.7 and 6.5 years) $(S D=2.94$ months) with a mean age of 73 months (6.1 years). The male-female ratio of the 34 participants without special needs was 19:15.

\subsection{PCS Recognition}

The aim of this study was to explore the effect of the number of symbol components on PCS recognition. Table 1 shows the words of the 30 PCSs. The vocabulary list was compiled according to a survey of vocabulary commonly used by kindergarten children. Each word had two corresponding PCS patterns with various numbers of components (a single symbol with one component and multiple symbols with five components). Table 2 shows an example of single and multiple symbols and the corresponding word. 
Table 1. Picture communication symbol (PCS) vocabulary list.

\begin{tabular}{|c|c|c|c|c|c|c|c|c|c|c|}
\hline Number & 1 & 2 & 3 & 4 & 5 & 6 & 7 & 8 & 9 & 10 \\
\hline Word & (Work) & (Play) & (Run) & (Give) & (Drink) & (Sleep) & (Please) & (Yes) & (Goodbye) & (No) \\
\hline Number & 11 & 12 & 13 & 14 & 15 & 16 & 17 & 18 & 19 & 20 \\
\hline Word & (Do) & (Eat) & (Go Home) & (Come) & (Punch) & (Use) & (Pull) & (Pat) & (Put) & (Wash) \\
\hline Number & 21 & 22 & 23 & 24 & 25 & 26 & 27 & 28 & 29 & 30 \\
\hline Word & (Wear) & (Take) & (Carry) & (Push) & (Paste) & (Buy) & (Write) & (Sell) & (Thank) & (Throw) \\
\hline
\end{tabular}

Table 2. PCS example and description.

\begin{tabular}{|c|c|c|}
\hline Pattern of Symbol & PCS (Give) & Component Description \\
\hline Single-symbol & & $\begin{array}{l}\text { 1. Made of one component } \\
\text { 2. A person extending one arm to depict the } \\
\text { movement of giving }\end{array}$ \\
\hline Multiple-symbol & "कू & $\begin{array}{l}\text { 1. Made of five components } \\
\text { 2. (a) A person extending one arm and to depict } \\
\text { the movement of giving (b) grasping an apple in } \\
\text { hand (c) directional arrow (d) a child (e) a table } \\
\text { full of apples (background) }\end{array}$ \\
\hline
\end{tabular}

\subsection{Research Instruments}

\subsubsection{Graphic Symbol Learning System}

This study aimed to objectively collect data regarding graphic symbol learning outcomes and to understand the factors of these learning outcomes. The research team conducted a literature review of published research and instructional experiences to design a graphic symbol learning software program as the primary research instrument in this study. The interactive teaching material was developed by Borland Delphi, which enabled the recording of user data in a database in a back-end system. The graphic symbol learning system is divided into two subsystems: Graphic Symbol Assessment and AAC graphic symbol animations. Figure 1 illustrates that the Graphic Symbol Assessment interface is divided into two portions. The top portion displays the question in written and audible forms (e.g., which one represents the "hitting" meaning?), whereas the bottom portion displays four clickable graphic symbols as answer options. In addition, user information and activity records are saved in the database of the learning system for subsequent uploading and analysis. Figure 2 illustrates that the Graphic Symbol animations interface is divided into two portions. The left portion includes the target word on top and the corresponding graphic symbol on the bottom, whereas the right portion displays the instruction combining texts, animation, and audio cues.

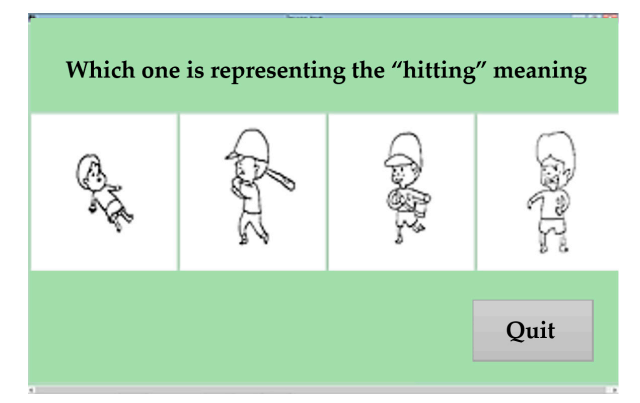

Figure 1. Screenshot of graphic symbol assessment. 


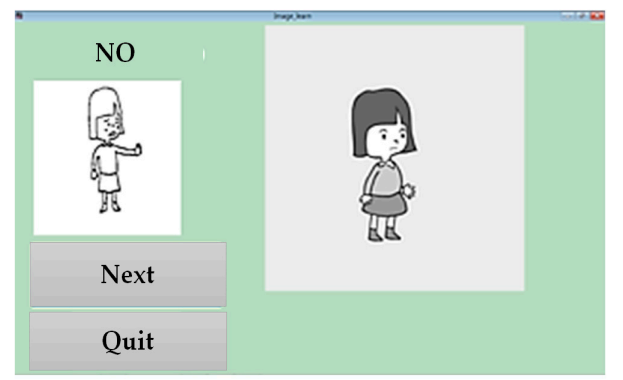

Figure 2. Screenshot of animations on graphic symbol vocabulary.

\subsubsection{MindWave EEG Device}

This study used the MindWave EEG headset developed by NeuroSky as the EEG detecting instrument. The headset employs ThinkGear technology to collect electronic signals from the brain and is as portable as a regular headset for music listening. In addition, the headset can be paired with a computer wirelessly through Bluetooth, and the device's user-friendliness favored the participants' usage and enabled the research team to collect the data in a natural environment. The electrodes are distributed in three touch points across the MindWave headset. In addition to detecting brainwaves in the frontal lobe, the frontal electrode also detects the user's blinking. The other two electrodes are normal electrodes that are primarily used to detect alpha and beta waves. Conductive adhesives are not required for the MindWave headset, leading to reduced test time by eliminating preparation.

\subsubsection{Liquid Crystal Display Touch Screen}

Because the participants' e-learning experience and computer literacy varied, this study employed a touch-screen interface for both PCS assessment and learning. A high-end computer featuring an independent graphic card, a four-core high-speed processor, and a liquid crystal display touch-screen monitor was used as the research instrument in this study to stabilize the clarity of graphic symbols, accelerate system response time, and minimize irrelevant factors.

\subsubsection{Real-Time EEG Monitoring System}

This study used the MindWave EEG headset developed by NeuroSky as the experimental instrument and combined it with a self-developed and patented real-time EEG monitoring system to obtain EEG data [47]. Once the headset had been successfully paired with the computer and had been properly donned by the participant, the EEG signal measurement was ready to start. The measured data in the headset were sent to the computer wirelessly through Bluetooth. The data were updated approximately once every second, and the updated data were immediately displayed on the program window on the computer screen. The EEG monitoring system displayed real-time alpha, beta, theta, and delta waves detected by the headset so that the research team could observe the experimental data of brainwave movement and obtain numerical values for variables such as attention, and relaxation parameters.

Before the real-time EEG monitoring system was turned on, the EEG headset was paired up with the computer. After the port value corresponding to the headset was confirmed, data collection began when the "Start" button was pressed. A brief waiting period followed while the headset was connecting to the system. Once the headset had paired with the system, the start menu was displayed on the screen. Once the user had clicked "OK" on the start menu, the EEG monitoring system began its monitoring process. The user was able to view the EEG data on the system menu while the monitoring system was running. Figure 3 illustrates that the top portion of the system screen displayed a line graph of the user's stress and energy indices while the bottom portion displayed a bar chart of various EEG band data. The screen refreshed once every second to monitor the user. In the top half of Figure 3, the line graph of stress and energy indices are displayed to enable comparison at each time point, 
whereas the bottom half displays real-time numerical changes of eight EEG bands comprising delta, theta, low alpha, high alpha, low beta, high beta, low gamma, and high gamma.

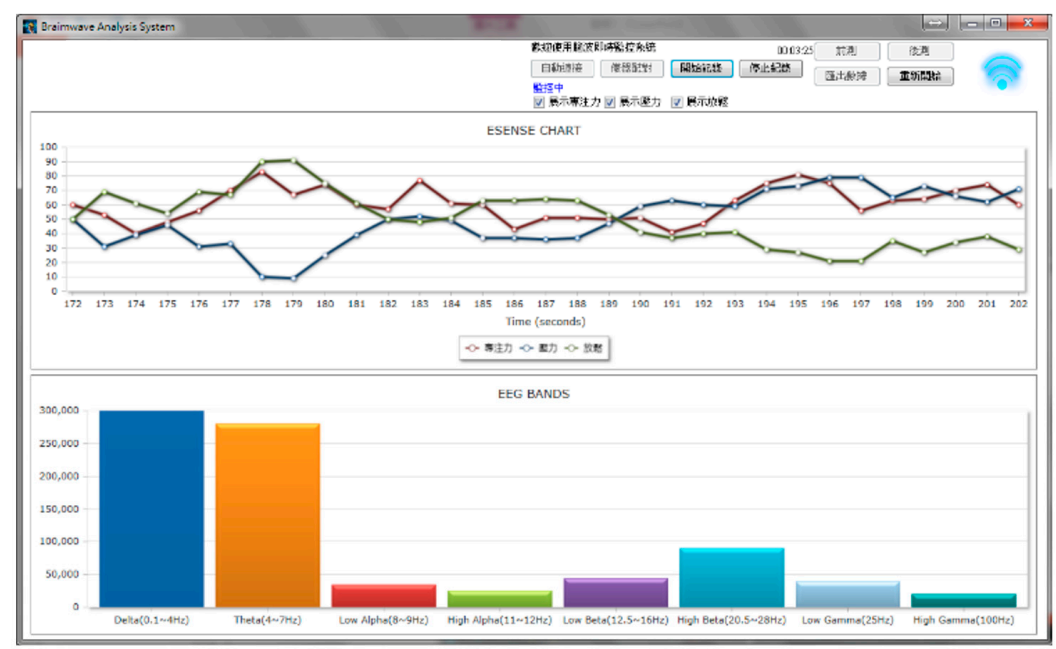

Figure 3. Screenshot of the real-time electroencephalogram (EEG) monitoring system.

The brainwave data detected from the MindWave headset consisted of eight EEG bands. However, because copious volumes of data were collected, the meanings of these brainwave data were difficult to understand without organization and analysis. Figure 4 illustrates the raw data measured by the monitoring system. The monitoring system converted raw data to an intuitive line chart and a bar chart, as shown in Figure 3. The researcher had to ensure that the MindWave EEG headset was worn on the participant's head properly once the real-time monitoring system had begun its recording. Subsequently, both the line chart and the bar chart appeared on the pretest and posttest monitoring screen. The three lines on the line chart on the top half of the screen comprised attention, stress, and relaxation parameters. Table 3 illustrates the frequency range of each EEG band. Recorded EEG raw data were filtered into different brainwaves. Before performing statistical analysis, the brainwave powers were subjected to a natural logarithm to make the distribution close to normality.

\begin{tabular}{|c|c|c|c|c|c|c|c|c|c|c|c|c|}
\hline \multicolumn{2}{|c|}{ Signal qual Attention } & \multicolumn{2}{|c|}{ Meditation Pressure } & Fatigue & Delta & \multirow{2}{*}{$\begin{array}{l}\text { Theta } \\
161857\end{array}$} & \multicolumn{2}{|c|}{ Low Alpba High Alph: } & Low Beta & \multirow{2}{*}{$\begin{array}{r}\text { High Beta } \\
8696\end{array}$} & \multicolumn{2}{|c|}{ Low Gamn High Gamma } \\
\hline 0 & 41 & 13 & 87 & 13 & 680353 & & 13749 & 9968 & 9949 & & 77439 & 9477 \\
\hline 0 & 54 & 30 & 70 & 9 & 1890389 & 610509 & 43025 & 82301 & 57048 & 57763 & 108906 & 37270 \\
\hline 0 & 43 & 43 & 57 & 2 & 193330 & 19201 & 7150 & 7234 & 2476 & 3519 & 1703 & 2289 \\
\hline 0 & 47 & 61 & 39 & 2 & 82572 & 9566 & 6018 & 998 & 622 & 107 & 1551 & 422 \\
\hline 0 & 37 & 48 & $\$ 2$ & 8 & 934054 & 97336 & 17678 & 6577 & 12227 & 14014 & 7109 & 17661 \\
\hline 0 & 48 & 53 & 47 & 5 & 1369723 & 152208 & 30410 & 25536 & 17028 & 32905 & 40424 & 19037 \\
\hline 0 & 53 & 26 & 74 & 38 & 3284521 & 735396 & 25481 & 12559 & 41514 & 92171 & 58876 & 79157 \\
\hline 0 & 67 & 29 & 71 & 1 & 238529 & 28711 & 30313 & 4395 & 807 & 11654 & 28738 & 9521 \\
\hline 0 & 63 & 37 & 63 & 6 & 591898 & 161774 & 25999 & 21688 & 7418 & 18195 & 10887 & 12394 \\
\hline 0 & 47 & 41 & 59 & 4 & $790 \mathrm{~m} 76$ & 143643 & 26247 & 32396 & 15194 & 10489 & 7336 & 10966 \\
\hline 0 & 48 & 66 & 34 & 3 & 126396 & 9989 & 2128 & 3255 & 670 & 2178 & 1712 & 828 \\
\hline 0 & 20 & 61 & 39 & 3 & 1018641 & 824781 & 213721 & 201288 & 60194 & 22381 & 122452 & 79195 \\
\hline 0 & 20 & 64 & 36 & 5 & 1812189 & 230481 & 69154 & 15381 & 2581 & 24278 & 20696 & 8401 \\
\hline 0 & 37 & 61 & 39 & s & 1545691 & 113489 & 15276 & 26349 & 20962 & 20992 & 16100 & 13190 \\
\hline 0 & 41 & 56 & 44 & 5 & 1002690 & 179667 & 18520 & 49694 & 14559 & 52952 & 76043 & 32590 \\
\hline 0 & 61 & 53 & 47 & 4 & 2600285 & 356548 & 20094 & 134512 & 24902 & 45779 & 22118 & 42313 \\
\hline 0 & 70 & 54 & 46 & 4 & 1702076 & 98053 & 14427 & 32171 & 2582 & 22604 & 12711 & 9567 \\
\hline 0 & 63 & 63 & 37 & 3 & 1268981 & 231154 & 98410 & 51288 & 24162 & 27832 & 60339 & 11462 \\
\hline 0 & 61 & 54 & 46 & 12 & 375316 & 72646 & 2136 & 9228 & 2498 & 13877 & 15013 & 9190 \\
\hline 0 & 60 & 35 & 65 & 25 & 258610 & 621311 & 27409 & 20477 & 36614 & 55282 & 48324 & 18999 \\
\hline 0 & 57 & 47 & 53 & 1 & 467605 & 43332 & 49296 & 23578 & 13588 & 16102 & 31742 & 15122 \\
\hline 0 & 66 & 51 & 49 & 3 & 1421142 & 471484 & 253565 & 15039 & 125177 & 121813 & 135784 & 30591 \\
\hline 0 & 88 & 63 & 37 & 5 & 1265541 & 217936 & 42106 & 32748 & 38814 & 126736 & 279911 & 52762 \\
\hline 0 & 78 & 66 & 34 & 1 & 2112888 & 437968 & 366027 & 440678 & 146266 & 81274 & 349463 & 81311 \\
\hline 0 & 69 & 54 & 46 & 17 & 796665 & 452374 & 28823 & 23358 & 9158 & 33318 & 92105 & 9341 \\
\hline 0 & 77 & 51 & 49 & 4 & 1110817 & 164909 & 40958 & 33366 & 14726 & 68444 & 19660 & 7903 \\
\hline
\end{tabular}

Figure 4. Screenshot of raw data in real-time EEG monitoring system. 
Table 3. Frequency range of EEG bands.

\begin{tabular}{cc}
\hline Brainwave & Range $\mathbf{( H z )}$ \\
\hline Delta & $0.1 \sim 4$ \\
Theta & $4 \sim 7$ \\
Low Alpha & $8 \sim 9$ \\
High Alpha & $11 \sim 12$ \\
Low Beta & $12.5 \sim 16$ \\
High Beta & $20.5 \sim 28$ \\
Low Gamma & 25 \\
High Gamma & 100 \\
\hline
\end{tabular}

\subsection{Procedure}

\subsubsection{EEG Headset Wearing and Calibration}

Before the formal pretest, posttest, or PCS instruction commenced, the participant was required to wear the EEG headset properly. After ensuring the participant was wearing the headset properly, the research team first interacted with the participant using a picture book. The purpose was to establish a relationship with the participant, mitigate the participant's unfamiliarity, and enable the participant to be accustomed to wearing a headset. Meanwhile, the headset was paired up with the computer and calibrated during the interaction between the research team and the participant. After the calibration was completed, the data collection was formally implemented.

\subsubsection{AAC Graphic Symbols Test}

The graphic symbols vocabulary test was conducted using the Graphic Symbol Assessment function of the Graphic Symbol Learning System. The test evaluated the participants' comprehension of the 30 aforementioned graphic symbols and the corresponding words. Because the participants were asked to wear the EEG headset and work with digital media and a tablet computer, the research staff arranged sufficient practice time before the formal pretest took place to mitigate irrelevant factors such as computer operation and headset wearing issues. The practice time enabled the participants to become familiar with the clicking and dragging methods on a touch-screen computer, and the participant's seating height and distance were also adjusted during the practice time. After the research team ensured that the participant was capable of understanding how to operate the computer and the learning system, the monitoring software was set up before the pretest commenced.

\subsection{Data Collection and Analysis}

This study collected the participants' identifying outcomes and brainwave histories for tests of AAC graphic symbols largely through the Graphic Symbol Learning System and EEG technology. To mitigate the effect of activities prior to the test on the participants' brainwave changes, all data collecting tests were conducted during kindergarten school hours after the lunch break. In addition, to prevent grading errors and irrelevant factors, the research team members served as evaluators. The assessment was carried out with touch-screen computers with identical specifications and a standardized oral guidance method. Once the participant put on the EEG headset properly, the research team spent 5-10 min talking to the participants casually and establishing a relationship to prevent evaluation errors caused by factors such as unfamiliarity and nervousness. Meanwhile, during this time span, the participant was allowed to become familiar with the clicking and dragging functions of the touch-screen computer. Subsequently, the research team operated the computer and the assessment software to display the assessment menu. The computer recorded the participant's answers and response times for questions containing various target words while the EEG headset and the real-time monitoring system recorded the participant's numerical brainwave data. This study used SPSS as a tool for statistical analysis. The mean and standard deviation were calculated for the accuracy 
of the participant's graphic symbols identification and for the EEG numerical values representing attention and relaxation parameters. The numerical values were paired up with graphs to illustrate the brainwave changes that reflected the participant's attention. Furthermore, this study conducted a two-way analysis of variance (ANOVA) to compare the effect and variance of the number of AAC graphic symbol components on identifying outcomes and brainwave histories of children with and without developmental delays. The integrated EEG Biofeedback function enabled the user to monitor his or her own attention for brainwave data analysis. Once the Graphic Symbol Learning System began running, the system automatically measured the EEG attentional performance as the baseline before the training. Subsequently, various degrees of attention during training were configured in the system from low (Attention: 50) to moderate (Attention: 70) and substantial (Attention: 90). Once the user put on the EEG headset, the EEG monitoring system performed real-time computation using the data measured from the MindWave headset and transmitted through the Bluetooth connection. In addition, the monitoring system calculated and displayed indices such as Pressure Index (PI), Meditation Index (MI), Body Energy Index (BEI), and Comprehensive Index (CI). These indices were calculated through the following equations:

PI can be converted from MI as follows:

$$
\mathrm{PI}=100-\beta \text { waves. }
$$

The Relaxation Index can be regarded as MI, and BEI can be calculated as follows:

$$
\mathrm{BEI}=\frac{(\text { High } \theta \text { waves }+ \text { Low } \theta \text { waves })}{2}
$$

CI can be calculated as follows:

$$
\mathrm{CI}=\theta \text { waves } /\left[\frac{(\text { High } \theta \text { waves }+ \text { Low } \theta \text { waves })}{2}\right]
$$

The Attention Index demonstrated the participant's mental focus or concentration. When the participant was able to control psychological activities in a highly concentrated state, the Attention Index (ranging between 0 and 100) increased. By contrast, mental states such as distraction, fainting, inattention, and anxiety decreased the Attention Index. The Relaxation Index, also ranging between 0 and 100, demonstrated the participant's mental calmness or relaxation. Several studies have confirmed that unstable brainwaves affect attention, thereby affecting the development of learning capacity. Therefore, the BCI in this study retrieved MindWave data through an EEG Biofeedback mechanism and uploaded the user's EEG Attention Index to a cloud server for statistical analysis of subsequent EEG attention training. According to the official data published by NeuroSky, the data accuracy of the MindWave headset is up to 90-96\% [48]. Other studies that have employed the MindWave headset have also confirmed that the headset has an accuracy up to $80 \%$ that facilitates a cost-effective, noninvasive brain machine interface for mind-controlling robotic arms by retrieving EEG signals [49]. Another study reported that EEG-based e-learning had a 96\% accuracy in detecting user's emotions [50].

\section{Experimental Results and Discussion}

\subsection{Success Rates of AAC Graphic Symbols Identification}

This study aimed to investigate the effect of a number of symbol components on graphic symbols recognition and learning outcomes. Table 4 shows the success rates of the children with and without developmental delays identifying the 30 graphic symbol sets with various numbers of components. Table 4 demonstrates that the average success rates for children with developmental delays identifying single- and multiple-component symbols were $37.04 \%(S D=17.03 \%)$ and $50.00 \%$ $(S D=17.09 \%)$, respectively. The overall success rate for these participants was $43.14 \%(S D=17.81 \%)$. By contrast, the average success rates for children without developmental delays identifying single- 
and multiple-component symbols were $64.71 \%(S D=15.23 \%)$ and $74.12 \%(S D=13.31 \%)$, respectively. The overall success rate for these participants was $69.41 \%(S D=14.87 \%)$. In addition, the overall success rates for the total population identifying single- and multiple-component symbols were $55.13 \%$ $(S D=20.53 \%)$ and $66.40 \%(S D=18.31 \%)$, respectively. This study conducted a two-way ANOVA to examine the effects of developmental status (i.e., children with developmental delays and peers) and a number of components (i.e., single- and multiple-component symbols) on the success rate of graphic symbols identification. The results revealed a nonsignificant interaction between developmental status and the number of components $(F=0.154, p>.05)$, indicating no interaction between these two variables. Subsequently, this study separately examined the main effects of these two variables. The first one-way ANOVA revealed a statistically significant interaction between developmental status and the success rate of graphic symbols identification $(F=32.656, p<0.05)$, indicating that the two population groups had a significant difference in graphic symbols identification. The mean numerical values also demonstrated that the children without developmental delays had a significantly higher success rate than did those with developmental delays. In addition, this study further explored the effect size of the developmental status variable on the success rate of graphic symbols identification. The results revealed that the developmental status variable could explain $41 \%$ of the variance of the success rate $\left(\eta^{2}=0.410\right)$. These two variables exhibited a strong correlation to each other [51]. By contrast, the second one-way ANOVA also revealed a statistical significance between a number of components and the success rate of graphic symbols identification $(F=6.096, p<0.05)$, indicating a significant difference for success rates between single- and multiple-component symbols. The mean numerical values also demonstrated that the participants had a significantly higher success rate at identifying multiple-component symbols than single-component symbols. The effect sizes for a number of components and success rates of graphic symbols identification indicated that the number of components explained $11.5 \%$ of the variance of the success rate $\left(\eta^{2}=0.115\right)$, indicating a moderate correlation between the two variables.

Table 4. Success rates of PCS identification for children with and without developmental delays (DD) (by percentage).

\begin{tabular}{|c|c|c|c|c|c|}
\hline Group & $N$ & $M$ & $S D$ & $F$ & $\eta^{2}$ \\
\hline $\begin{array}{l}\text { DD_single-symbol } \\
\text { multiple-symbol }\end{array}$ & 17 & $\begin{array}{l}37.04 \\
50.00\end{array}$ & $\begin{array}{l}17.03 \\
17.09\end{array}$ & & \\
\hline $\begin{array}{l}\text { Peers-single-symbol } \\
\text { multiple-symbol }\end{array}$ & 34 & $\begin{array}{l}64.71 \\
74.12\end{array}$ & $\begin{array}{l}15.23 \\
13.31\end{array}$ & & \\
\hline DD (All) & 17 & 43.14 & 17.81 & \multirow{2}{*}{32.656 * } & \multirow{2}{*}{0.410} \\
\hline Peers (All) & 34 & 69.41 & 14.87 & & \\
\hline Single-symbol (All) & 51 & 55.13 & 20.53 & \multirow{2}{*}{6.096 * } & \multirow{2}{*}{0.115} \\
\hline Multiple-symbol (All) & 51 & 66.40 & 18.31 & & \\
\hline
\end{tabular}

\subsection{The Variance of Attention Index}

This study investigated the effect of a number of components on participants' EEG differences when they attempted to identify graphic symbol sets. Table 5 shows the average Attention Index values of the children with and without developmental delays identifying the 30 graphic symbol sets with various numbers of components. Table 5 demonstrates that the average Attention Index values for children with developmental delays identifying single- and multiple-component symbols were $60.22(S D=14.66)$ and $50.00(S D=7.29)$, respectively. The overall Attention Index for these participants was $55.41(S D=12.59)$. By contrast, the average Attention Index values for children without developmental delays identifying single- and multiple-component symbols were 50.33 $(S D=11.13)$ and $45.16(S D=7.76)$, respectively. The overall Attention Index for these participants was 
$47.44(S D=9.60)$. In addition, the overall Attention Index values for the total population identifying single- and multiple-component symbols were $54.04(S D=13.19)$ and $46.59(S D=7.82)$, respectively. The research team conducted a two-way ANOVA to examine the effects of developmental status and the number of components on the Attention Index during graphic symbols identification. The results revealed a nonsignificant interaction between developmental status and a number of components $(F=0.686, p>0.05)$, indicating no interaction between these two variables. Subsequently, this study separately examined the main effects of these two variables. The first one-way ANOVA revealed a statistical significance between developmental status and Attention Index $(F=5.845, p<0.05)$, indicating that the two population groups had a significant difference in Attention Index. The mean numerical values also demonstrated that the children with developmental delays had significantly higher Attention Index values than those without developmental delays did. In addition, this study further explored the effect size of the developmental status variable on the Attention Index. The results revealed that the developmental status variable could explain $11.1 \%$ of the variance of Attention Index $\left(\eta^{2}=0.111\right)$. According to Cohen [51], these two variables exhibited a moderate correlation with each other. By contrast, the second one-way ANOVA also revealed a statistical significance between a number of components and Attention Index $(F=6.386, p<0.05)$, indicating the significant difference in Attention Index between single- and multiple-component symbols. The mean numerical values also demonstrated that the participants had a significantly higher Attention Index on single-component symbols than multiple-component symbols. The effect size between a number of components and Attention Index indicated that the number of components explained $12 \%$ of the variance of the index $\left(\eta^{2}=0.120\right)$, indicating a moderate correlation between the two variables.

Table 5. Attention Index of children with and without developmental delays (DD) during PCS identification.

\begin{tabular}{|c|c|c|c|c|c|}
\hline Group & $N$ & $M$ & $S D$ & $F$ & $\eta^{2}$ \\
\hline $\begin{array}{l}\text { DD_single-symbol } \\
\text { multiple-symbol }\end{array}$ & 17 & $\begin{array}{l}60.22 \\
50.00\end{array}$ & $\begin{array}{l}14.66 \\
7.29\end{array}$ & & \\
\hline $\begin{array}{l}\text { Peer-single-symbol } \\
\text { multiple-symbol }\end{array}$ & 34 & $\begin{array}{l}50.33 \\
45.16\end{array}$ & $\begin{array}{l}11.13 \\
7.76\end{array}$ & & \\
\hline DD (All) & 17 & 55.41 & 12.59 & \multirow{2}{*}{5.845 * } & \multirow{2}{*}{0.111} \\
\hline Peer (All) & 34 & 47.44 & 9.60 & & \\
\hline Single-symbol (All) & 51 & 54.04 & 13.19 & \multirow{2}{*}{$6.386^{*}$} & \multirow{2}{*}{0.120} \\
\hline Multiple-symbol (All) & 51 & 46.59 & 7.82 & & \\
\hline
\end{tabular}

\subsection{The Variance of Relaxation Index}

Table 6 shows the average Relaxation Index values of the children with and without developmental delays identifying the 30 graphic symbol sets with various numbers of components. Table 6 demonstrates that the average Relaxation Index values for children with developmental delays identifying single- and multiple-component symbols were $55.56(S D=8.23)$ and $61.88(S D=9.06)$, respectively. The overall Relaxation Index for these participants was 58.53 ( $S D=8.97)$. By contrast, the average Relaxation Index values for children without developmental delays identifying single- and multiple-component symbols were $60.07(S D=10.98)$ and $59.37(S D=9.67)$, respectively. The overall Relaxation Index for these participants was $59.68(S D=10.11)$. In addition, the overall Relaxation Index values for the total population identifying single- and multiple-component symbols were 58.37 $(S D=10.10)$ and $60.11(S D=9.67)$, respectively. The research team conducted a two-way ANOVA to examine the effects of developmental status and the number of components on the Relaxation Index during graphic symbols identification. The results revealed a nonsignificant interaction between developmental status and the number of components $(F=1.451, p>0.05)$, indicating no interaction 
between these two variables. Subsequently, this study separately examined the main effects of these two variables. However, no significant differences were observed in a one-way ANOVA between developmental status and Relaxation Index $(F=0.118, p>0.05)$; no significant differences were observed in a one-way ANOVA between a number of components and Relaxation Index $(F=0.931$, $p>0.05)$. Therefore, no variance of Relaxation Index was yielded by these two variables during graphic symbols identification.

Table 6. Relaxation Index of children with and without developmental delays (DD) during PCS identification.

\begin{tabular}{|c|c|c|c|c|}
\hline Group & $N$ & $M$ & $S D$ & $F$ \\
\hline $\begin{array}{l}\text { DD_single-symbol } \\
\text { multiple-symbol }\end{array}$ & 17 & $\begin{array}{l}55.56 \\
61.88\end{array}$ & $\begin{array}{l}8.23 \\
9.06\end{array}$ & \\
\hline $\begin{array}{l}\text { Peers-single-symbol } \\
\text { multiple-symbol }\end{array}$ & 34 & $\begin{array}{l}60.07 \\
59.37\end{array}$ & $\begin{array}{c}10.98 \\
9.67\end{array}$ & \\
\hline DD (All) & 17 & 58.53 & 8.97 & \multirow{2}{*}{0.118 (n.s.) } \\
\hline Peers (All) & 34 & 59.68 & 10.11 & \\
\hline Single-symbol (All) & 51 & 58.37 & 10.10 & \multirow{2}{*}{0.931 (n.s.) } \\
\hline Multiple-symbol (All) & 51 & 60.11 & 9.39 & \\
\hline
\end{tabular}

\section{Conclusions}

The purpose of this study was to investigate by EEG the influence of symbol component on the recognition of AAC graphic symbols for kindergarten children with and without developmental delays. The results of this study revealed that the children without developmental delays had a more favorable performance in graphic symbols identification than those with developmental delays did, and both populations had a higher success rate at identifying multiple-component symbols than single-component symbols. Therefore, the multiple-component symbols were more easily identified than the single-component symbols were, indicating a positive relationship between the number of components and iconicity. The results of this study are similar to those of previous studies, which confirm that a high number of constituent elements of graphics symbols $[13,16,19]$ or constitute more complex graphic symbols [3,52] is more conducive to children's identification of graphic symbols. This result lends only limited support to the visual stimuli proposed by Paivio's [24] dual-coding theory, which for readers is conducive to forming a coherent mental representation and identification of graphic symbols.

The results also demonstrated that the children with developmental delays had a significantly higher Attention Index than those without developmental delays did during graphic symbols identification. Although the children with developmental delays had a relatively low success rate at graphic symbols identification, they paid more attention during graphic symbols identification than the children without developmental delays did. The participants collectively had a significantly higher Attention Index for single-component symbols than for multiple-component symbols, indicating that the graphic symbols made of multiple components could not facilitate the participants' reading focus. From the perspective of cognitive load theory, multiple stimuli probably decreased the participants' reading focus. The aforementioned research findings demonstrated that the children without developmental delays initially had a significantly higher success rate at identifying AAC graphic symbols than those with developmental delays did. The children with developmental delays had a lower Attention Index than those without developmental delays did. The opposite outcomes between the success rate and Attention Index suggests that the initial iconicity was not correlated to Attention Index, and the participants' cognitive capacity apparently had a stronger effect on success rate than Attention Index did. Although the average rate of recognition of children with developmental delays is lower than typically developing children, the Attention Index of identifying graphic symbols 
is higher. According to the above results, the authors concluded that children with developmental delays may be limited to moderate and severe cognitive function. When there are too few clues leading to graphic symbols, the process of using cognitive thinking in identifying graphic symbols is relatively brief and characterized by guessing. Therefore, in children with developmental delays, the success rate of recognition is lower and the Attention Index is higher, while typically developing children show the opposite trend.

In addition, the participants had a significantly higher success rate at identifying multiple-component symbols than single-component symbols, but their Attention Index average was higher when reading single-component symbols than when reading multiple-component symbols. Essentially, although a high number of symbol components decreased the participants' average Attention Index, the multiple-component symbols were more easily recognized and identified than the single-component symbols were. The results show that the relationship between the success rate of recognition and the Attention Index is inversely proportional. This finding also confirmed cognitive load theory, which predicts that a cognitive overload affects the participants' reactions $[25,26]$.

This study is limited to research media because each graphic symbol requires a short reading time, so it is more difficult to effectively present the influence on brainwaves and on concentration. In the future, a picture book can be used as a reading medium so that the influence on brainwaves and concentration in pattern recognition, as well as the processes children use when reading a page of graphic aids and when reading a page of text, can be examined. In teaching practice as well as in AAC practice, we can refer to these research findings and provide graphic symbolic media composed of multiple elements to promote the identification and learning of graphic symbols in children with developmental delays.

Author Contributions: Methodology, C.-H.H. and P.-J.L.; Project administration, C.-H.H.; Software, P.-J.L.; Writing —original draft, C.-H.H. and P.-J.L.; Writing—review \& editing, P.-J.L. and C.-H.H.

Acknowledgments: The authors would like to thank the Ministry of Science and Technology of the Republic of China, Taiwan, for financially supporting this research under Contract Nos. MOST 104-2221-E-241-012-MY2 and MOST 107-2511-H-241-001.

Conflicts of Interest: The authors declare no conflict of interest.

\section{References}

1. Barker, R.M.; Akaba, S.; Brady, N.C.; Thiemann-Bourque, K. Support for AAC use in preschool and growth in language skills for Young Children with Developmental Disabilities. Augment. Altern. Commun. 2013, 29, 334-346. [CrossRef]

2. de Almeida Barbosa, R.T.; de Oliveira, A.S.; de Lima Antão, J.Y.; Crocetta, T.B.; Guarnieri, R.; Antunes, T.P.; Arab, C.; Massetti, T.; Bezerra, I.M.; de Mello Monteiro, C.B.; et al. Augmentative and alternative communication in children with Down's syndrome: A systematic review. BMC Pediatr. 2018, 18, 160. [CrossRef]

3. Mahoney, B.; Johnson, A.; McCarthy, M.; White, C. Systematic Review: Comparative Efficacy of the Picture Exchange Communication System (PECS) to Other Augmentative Communication Systems in Increasing Social Communication Skills in Children with Autism Spectrum Disorder. In Communication Sciences and Disorders: Systematic Review Publications; The University of Vermont: Burlington, VT, USA, 2018.

4. Mukhopadhyay, S.; Nwaogu, P. Barriers to teaching non-speaking learners with intellectual disabilities and their impact on the provision of augmentative and alternative communication. Int. J. Disabil. Dev. Educ. 2009, 56, 349-362. [CrossRef]

5. Beukelman, D.R.; Mirenda, P. Augmentative and Alternative Communication: Support Children and Adults with Complex Communication Needs, 4th ed.; Paul H. Brooks: Baltimore, MD, USA, 2013.

6. Emms, L.; Gardner, H. Study of two graphic symbol-teaching methods for individuals with physical disabilities and additional learning difficulties. Child Lang. Teach. Ther. 2010, 2, 5-22. [CrossRef]

7. Karal, Y.; Karal, H.; Silbir, L.; Altun, T. Standardization of a graphic symbol system as an alternative communication tool for Turkish. Educ. Technol. Soc. 2016, 19, 53-66. 
8. Schlosser, R.W.; Koul, R.; Shane, H.; Sorce, J.; Brock, K.; Harmon, A.; Moerlein, D.; Hearn, E. Effects of animation on naming and identification across two graphic symbol sets representing verbs and prepositions. J. Speech Lang. Hear. Res. 2014, 57, 1779-1791. [CrossRef]

9. Tönsing, K.M.; Dada, S.; Alant, E. Teaching graphic symbol combinations to children with limited speech during shared story reading. Augment. Altern. Commun. 2014, 30, 279-297. [CrossRef] [PubMed]

10. Dada, S.; Huguet, A.; Bornman, J. The iconicity of picture communication symbols for children with English additional language and mild intellectual disability. Augment. Altern. Commun. 2013, 29, 360-373. [CrossRef]

11. Nam, S.; Kim, J.; Sparks, S. An Overview of Review Studies on Effectiveness of Major AAC Systems for Individuals with Developmental Disabilities Including Autism. J. Spec. Educ. Apprenticesh. 2018, 7, n2.

12. Graves, M.F.; Watts-Taffe, S. For the love of words: Fostering word consciousness in young readers. Read. Teach. 2008, 62, 185-193. [CrossRef]

13. Huang, C.H. Influence of symbol components on the understanding of verb graphic symbols of preschool children with and without developmental delay. NTTU Educ. Res. J. 2017, 28, 31-59. [CrossRef]

14. Lemons, C.J.; King, S.A.; Davidson, K.A.; Puranik, C.S.; Al Otaiba, S.; Fidler, D.J. Personalized reading intervention for children with Down syndrome. J. Sch. Psychol. 2018, 66, 67-84. [CrossRef]

15. Sandall, S.R.; Schwartz, I.S. Building Blocks for Teaching Preschoolers with Special Needs; Paul H. Brookes Publishing Co.: Baltimore, MD, USA, 2002.

16. Alant, E.; Life, H.; Harty, M. Comparison of learnability and retention between Blissymbols and CyberGlyphs. Int. J. Lang. Commun. Disord. 2005, 40, 151-169. [CrossRef]

17. Angermeier, K.; Schlosser, R.W.; Luiselli, J.K.; Harrington, C.; Carter, B. Effects of iconicity on requesting with the picture exchange communication system in children with autism spectrum disorder. Res. Autism Spectr. Disord. 2008, 2, 430-446. [CrossRef]

18. Huang, C.H.; Chen, M.C. Effect of translucency on transparency and symbol learning for children with and without cerebral palsy. Res. Dev. Disabil. 2011, 32, 1829-1836. [CrossRef]

19. Mizuko, M.; Reichle, J. Transparency and recall of symbols among intellectually handicapped adults. J. Speech Hear. Disord. 1989, 54, 627-633. [CrossRef]

20. Lloyd, L.L.; Fuller, D.R. The Role of Iconicity in Augmentative and Alternative Communication Symbol Learning. In Key Issues in Mental Retardation Research; Fraser, W.I., Ed.; Routledge: London, UK, 1990.

21. Schlosser, R.W.; Sigafoos, J. Selecting graphic symbols for an initial request lexicon: Integrative review. Augment. Altern. Commun. 2002, 18, 102-123. [CrossRef]

22. Luftig, R.L.; Bersani, H.A. An initial investigation of translucency, transparency, and component complexity of Blissymbolics. J. Child. Commun. Disord. 1985, 8, 191-209. [CrossRef]

23. Eysenck, M.W.; Keane, M.T. Cognitive Psychology: A Student's Handbook, 4th ed.; Psychology Press: Hove, UK, 2000.

24. Paivio, A. Dual coding theory. In Mental Representations: A Dual Coding Approach; Paivio, A., Ed.; Oxford University Press: New York, NY, USA, 1992; pp. 53-83.

25. Sweller, J. Implications of cognitive load theory for multimedia learning. In The Cambridge Handbook of Multimedia Learning; Mayer, R.E., Ed.; Cambridge University Press: New York, NY, USA, 2005; pp. 19-30.

26. Kalyuga, S. Cognitive Load Theory: How Many Types of Load Does It Really Need? Educ. Psychol. Rev. 2011, 23, 1-19. [CrossRef]

27. Huang, C.H.; Chen, M.C. Exploring the translucency of picture communication symbols for important adults of children with cerebral palsy in regular classes. J. Spec. Educ. 2011, 33, $29-56$.

28. Mirenda, P.; Locke, P.A. A Comparison of Symbol Transparency in Nonspeaking Persons with Intellectual Disabilities. J. Speech Hear. Disord. 1989, 54, 131-140. [CrossRef]

29. Thistle, J.J.; Holmes, S.A.; Horn, M.M.; Reum, A.M. Consistent Symbol Location Affects Motor Learning in Preschoolers Without Disabilities: Implications for Designing Augmentative and Alternative Communication Displays. Am. J. Speech-Lang. Pathol. 2018, 27, 1010-1017. [CrossRef]

30. Raj, A.; Jaisakthi, S.M. Analysis of Brain Wave Due to Stimulus Using EEG. In Proceedings of the 2018 International Conference on Computer, Communication, and Signal Processing (ICCCSP), Chennai, India, 22-23 February 2018; pp. 1-4.

31. Taksande, S.; Padole, D.V. Brain machine interface system for person with quadriplegia disease. In. J. Comput. Appl. Technol. Res. 2014, 3, 339-344. 
32. Campbell, J.I.; Fugelsang, J. Strategy choice for arithmetic verification: Effects of numerical surface form. Cognition 2001, 80, 21-30. [CrossRef]

33. Yeh, T.L.; Chen, C.C. A study of e-picture books and traditional picture books on children's reading attention. In Advances in Physical Ergonomics and Human Factors; Springer International Publishing: Cham, Switzerland, 2016; pp. 635-642.

34. Lin, C.S.; Yang, H.J.; Lay, Y.L.; Yang, S.W.; Liou, S.R. The special design and use intention evaluation of a brain wave measuring and learning system for physical installation in the museum. Indian J. Fundam. Appl. Life Sci. 2012, 2, 145-159.

35. Kim, J.H.; Chung, E.J.; Lee, B.H. A study of analysis of the brain wave with respected to action observation and motor imagery: A pilot randomized controlled trial. J. Phys. Ther. Sci. 2013, 25, 779-782. [CrossRef]

36. Hurless, N.; Mekic, A.; Pena, S.; Humphries, E.; Gentry, H.; Nichols, D. Music genre preference and tempo alter alpha and beta waves in human non-musicians. Prem. Undergrad. Neurosci. J. 2013, 1, 1-11.

37. Shin, M.C.; Jung, K.S.; Choi, Y.S. Learning contents type recommendation using profile in emotion based interactive e-learning environment. J. Comput. Sci. Eng. 2012, 39, $25-32$.

38. Shen, L.; Wang, M.; Shen, R. Affective e-learning: Using "Emotional" data to improve learning in pervasive learning environment. Educ. Technol. Soc. 2009, 12, 176-189.

39. Hosseini, S.; Maleki, I.; Farnia, S.; Kazemi-Malekmahmoudi, S. Comparing the effectiveness of music therapy and alpha-theta neuro-feedback training on anxiety and depression among patients with chronic irritable bowel syndrome. Chronic Dis. J. 2018, 6, 143-151.

40. Klimesch, W.; Doppelmayr, M.; Wimmer, H.; Gruber, W.; Röhm, D.; Schwaiger, J.; Hutzler, F. Alpha and beta band power changes in normal and dyslexic children. Clin. Neurophysiol. 2001, 112, 1186-1195. [CrossRef]

41. Jung, K.S.; Choi, Y.S. Brain wave and user profile based learning content type recommendation in interactive e-learning environment. Int. J. Smart Home 2012, 6, 33-40.

42. Ubilluz, C.; Delgado, R.; Marcillo, D.; Noboa, T. Brain Waves Processing, Analysis and Acquisition to Diagnose Stress Level in the Work Environment. Trends Adv. Inf. Syst. Technol. 2018, 859-866.

43. Dev, A.; Rahman, M.A.; Mamun, N. Design of an EEG-Based Brain Controlled Wheelchair for Quadriplegic Patients. In Proceedings of the 2018 3rd International Conference for Convergence in Technology (I2CT), Pune, India, 6-8 April 2018; pp. 1-5.

44. Lee, S.; Shin, Y.; Woo, S.; Kim, K.; Lee, H.-N. Review of wireless brain-computer interface systems. In Brain-Computer Interface-Recent Progress and Future Prospects; InTech: Rijeka, Croatia, 2013; pp. 216-238.

45. Bozkurt, F.; Coskun, H.; Aydogan, H. Effectiveness of classroom lighting colors toward students' attention and meditation extracted from brainwave. J. Educ. Instruct. Stud. World 2014, 4, 6-12.

46. Männel, C.; Schaadt, G.; Illner, F.K.; van der Meer, E.; Friederici, A.D. Phonological abilities in literacy-impaired children: Brain potentials reveal deficient phoneme discrimination, but intact prosodic processing. Dev. Cogn. Neurosci. 2017, 23, 14-25. [CrossRef]

47. Lin, P.J.; Chen, Y.Q.; Wu, J.H.; Xu, M.S. Brain training system using Electroencephalography (EEG) technology. In Proceedings of the 37th Annual International Conference of the IEEE Engineering in Medicine and Biology Society (EMBC 2015), Milano, Italy, 25-29 August 2015.

48. Neurosky. NeuroskyMindwave. 2015. Available online: http://neurosky.com/resources/ (accessed on 5 August 2015).

49. Salvekar, D.; Nair, A.; Bright, D.; Bhisikar, S.A. Mind controlled robotic arm. IOSR J. Electron. Commun. Eng. 2015, 54, 36-44.

50. Prabhu, N.G.; Singh, N.S.; Singh, S.V.; Patil, N. Affective e-Learning using emotion detection. Int. J. Tech. Res. Appl. 2016, 4, 216-220.

51. Cohen, J. Statistical Power Analysis for the Behavioral Sciences, 2nd ed.; Lawrence Erlbaum Associates: Hillsdale, NJ, USA, 1988.

52. Fuller, D.R. Initial study into the effects of translucency and complexity on the learning of Blissymbols by children and adults with normal cognitive abilities. Augment. Altern. Commun. 1997, 13, 30-39. [CrossRef]

(C) 2019 by the authors. Licensee MDPI, Basel, Switzerland. This article is an open access article distributed under the terms and conditions of the Creative Commons Attribution (CC BY) license (http:// creativecommons.org/licenses/by/4.0/). 